\title{
Crus Helicis
}

National Cancer Institute

\section{Source}

National Cancer Institute. Crus Helicis. NCI Thesaurus. Code C32410.

The external rim of the auricula (ear). 